\title{
Spontaneous rupture of the oesophagus:

\author{
Is conservative treatment ever justified?
}

\author{
S. MOVSA S ${ }^{1}$ \\ From the Department of Surgery, University of Natal, South Africa
}

As long ago as 1724, Boerhaave published the first description of spontaneous rupture of the oesophagus. His 70-page article contains the full clinical details and the findings at the necropsy of his patient Baron von Wassenaer, Grand Admiral of the Dutch Fleet. Despite this classical description, 134 years passed before Meyer, in 1858, first reported the condition diagnosed while the patient was still alive. Even after this, the frequent demonstration at necropsy of many cases missed clinically attest to the diagnostic difficulties. Nevertheless, in recent years rupture of the oesophagus has been correctly recognized soon after onset with increasing frequency. With earlier diagnosis, more and more patients have undergone thoracotomy with repair of the oesophageal tear. Such treatment, first successfully performed by Barrett in 1947, provides the best chance of survival. Without thoracotomy, almost without exception, patients succumb. The following is a description of such a rare exception.

\section{CASE REPORT}

On 16 July 1963, C. M., an Indian man aged 46 years, was admitted to King Edward VIII Hospital. He was very restless and spoke so incoherently between rapid grunting respirations $(44 / \mathrm{min}$.) that it was impossible to obtain a history. However, a relative stated that six hours before admission he had vomited a cupful of blood and immediately thereafter had complained of severe epigastric and substernal pain. When first examined the patient's temperature was normal. His pulse rate was $120 / \mathrm{min}$. and his blood pressure $140 / 90 \mathrm{~mm}$. Hg. Abdominal examination revealed generalized tenderness and rigidity. Bowel sounds were absent. No abnormality was detected per rectum. On auscultation of the chest crepitations were heard over both lung bases. The root of the neck was not palpated for evidence of subcutaneous emphysema. The haemoglobin was $15.3 \mathrm{~g} . / 100 \mathrm{ml}$, leucocyte count $13,000 /$ c.mm., and serum amylase 8 Wohlgemuth units. Perforation of a peptic ulcer was diag-

1Present address: University of Minnesota Medical School, Minneapolis, Minnesota 55455 nosed and a laparotomy was performed. Notwithstanding a gastrotomy, no abnormality was detected in the stomach or duodenum. The other abdominal viscera were also normal.

Two days after admission subcutaneous emphysema was detected at the root of the neck. The presence of gas in the tissue planes of the neck was confirmed by a chest radiograph which also showed widening of the inferior mediastinum and consolidation of the right apical lobe. At this stage spontaneous rupture of the oesophagus was diagnosed. In view of the patient's good general condition and as there was neither air nor liquid in the pleural cavity, it was decided to continue conservative treatment. Over the next three days the patient's temperature ranged between 97 and $100^{\circ} \mathrm{F}$., the respiratory rate averaged $30 / \mathrm{min}$., and the pulse ranged from 80 to $100 / \mathrm{min}$. On the sixth day after admission a chest radiograph revealed a small effusion in the right pleural cavity and consolidation of the right lung base. A gastrografin swallow showed an irregular area at the lower end of the oesophagus with extravasation of contrast medium into the para-oesophageal tissue (Fig. 1), confirming rupture of the oesophagus. To exclude an underlying oesophageal ulcer or carcinoma it was decided to perform an oesophagoscopy. During induction of anaesthesia for this procedure the patient vomited and aspirated gastric contents into the bronchial tree. The oesophagoscopy was abandoned. Instead a bronchoscope was passed and the main bronchi were cleared. A tracheostomy was then performed. On the patient's return to the ward, it was noticed that dehiscence of the abdominal wound had occurred. As skin apposition was maintained by the still intact skin sutures, and as the patient's general condition was very poor, it was decided not to repair the dehiscence. Instead the abdominal wound was reinforced by an adhesive plaster corset.

During the following week the patient's condition steadily improved. Jejunostomy was performed and feeding was started by this route two weeks after admission. A chest radiograph showed very little fluid at the right lung base. The patient was now apyrexial. One month after admission a barium swallow (Fig. 2) failed to reveal any extravasation of radio-opaque material from the oesophagus, which had completely healed. This was confirmed a few days later by oesophagoscopy which showed only a slightly yellow area, presumably due to a healed scar, 


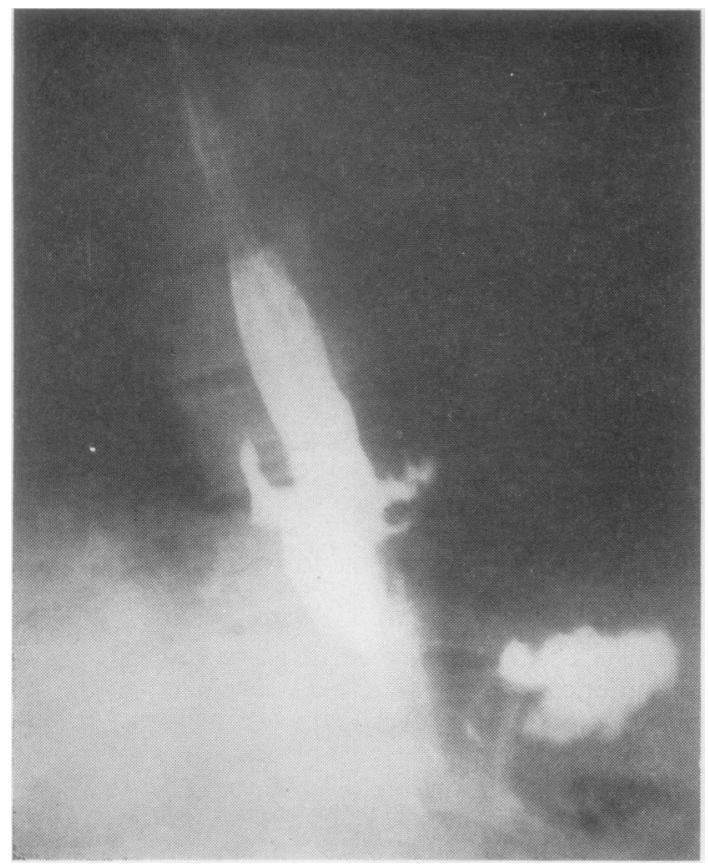

FIG. 1. Gastrografin swallow showing extravasation of dye from lower end of oesophagus six days after spontaneous rupture.

in the region where the oesophageal wall had ruptured. Oral fluids were started and taken well. On 25 August 1963, nearly six weeks after admission, the patient was discharged from hospital. swallowing without difficulty.

Subsequently the patient was seen three months, six months, and just over a year later. On each of these occasions he was symptom free. He stated that he was swallowing well and had not lost weight. On examination he was found to have an incisional hernia, for which he refused operative repair. Nearly a year after the patient's discharge from hospital, a barium swallow was carried out. The anterior wall of the lower end of the oesophagus showed a consistent deformity without mucosal destruction, suggesting scarring and fibrosis of this region of the oesophagus (Fig. 3). A small amount of gastrooesophageal reflux occurred.

\section{DISCUSSION}

The clinical diagnosis of spontaneous rupture of the oesophagus may be difficult. Nevertheless the picture is now becoming well known and distinctive enough to be increasingly recognized. Thus although Barrett in 1947 had collected only a little over 50 cases of spontaneous oesophageal rupture, Moynihan, by 1954, had traced more than 200 published cases. Characteristically, obese

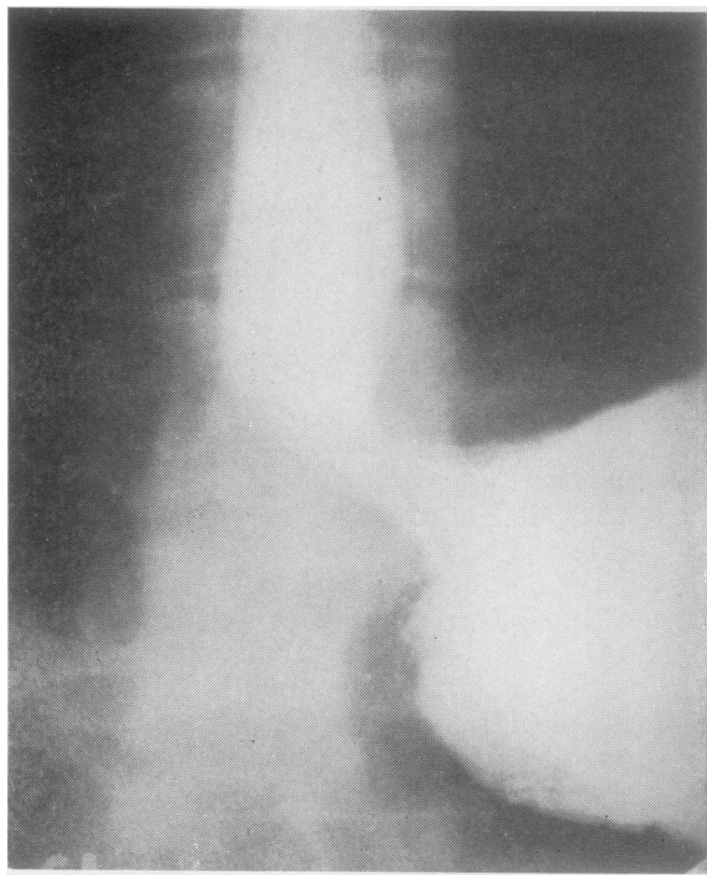

FIG. 2. Barium swallow four weeks after spontaneous rupture of the oesophagus. Complete healing of oesophageal tear has occurred.

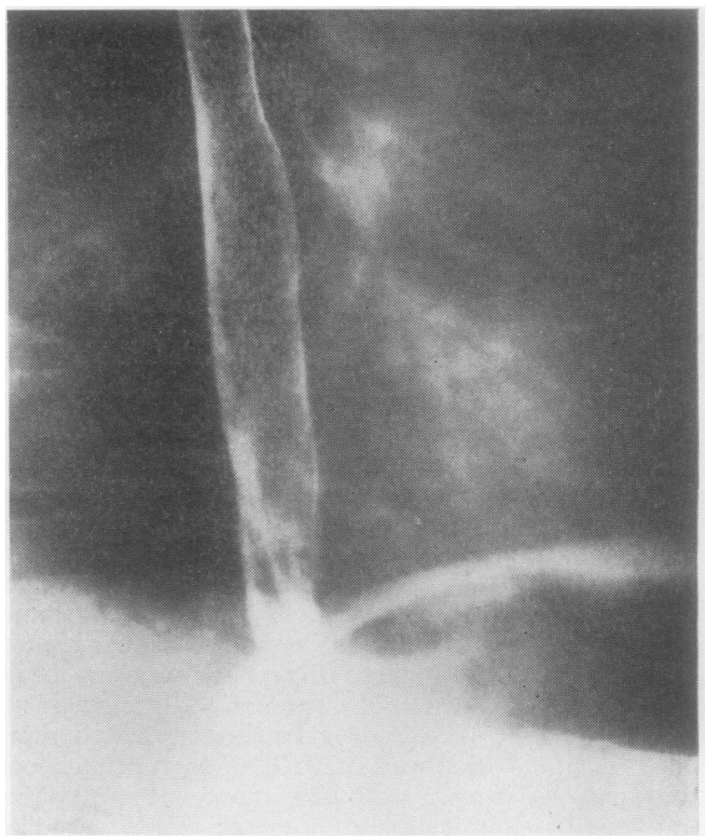

FIG. 3. Barium swallow showing minimal deformity due to scarring of lower end of oesophagus one year after spontaneous rupture. 
middle-aged men are affected nine times as frequently as women (Storey, 1962). Following a bout of vomiting, the patient is usually seized with a sudden violent epigastric or precordial pain aggravated by swallowing or breathing. Haematemesis is rare and when it does occur, as in this patient, it is often mild. This is in sharp contrast to the severe bouts of haematemesis, so prominent a feature of mucosal tears at the oesophago-gastric junction (Mallory and Weiss, 1929). Thirst is often an outstanding symptom, and dyspnoea, cyanosis, and rapid grunting respirations commonly occur. Epigastric tenderness and rigidity may lead to the erroneous diagnosis of perforated peptic ulcer or acute pancreatitis. The presence of subcutaneous emphysema at the root of the neck, found in $65 \%$ of cases (Tesler and Eisenberg, 1963), strongly suggests oesophageal rupture. Radiological examination of the chest reveals a pleural effusion or hydropneumothorax in $91 \%$ of cases and mediastinal emphysema in $66 \%$ (Priviteri and Gay, 1951). In such patients the diagnosis is no longer in doubt. For the more difficult case, where the diagnosis is suspected but not definite, a radiograph after swallowing radio-opaque dye will supply conclusive evidence that the oesophagus is ruptured.

In the patient here reported the correct diagnosis was initially missed. Instead, perforation of a peptic ulcer, a quite common condition among our Indian patients, was suspected but could not be confirmed at laparotomy. It was not until two days after admission, when surgical emphysema at the root of the neck was first noted, that the correct diagnosis was made. Thus at this stage the problem arose how best further to treat the patient. Three methods were considered: thoracotomy with repair of the oesophageal tear, thoracotomy and drainage of the left pleural cavity, and the continuation of non-operative treatment.

Previous reports in the literature were searched for guidance. Almost every review stressed that the prognosis was hopeless without operation. Thus Kinsella, Morse, and Hertzog (1948) found that only eight of 53 reported cases of spontaneous rupture of the oesophagus not operated on survived longer than 48 hours, while Eliason and Welty (1946) reported a mortality of $100 \%$ for such cases. However, Graham (1944) mentioned two patients who survived following drainage of the pleural cavity without suture of the oesophageal tear. A similar case was reported by Moore and Murphy (1948). In their patient the diagnosis was initially missed. On admission, a laparotomy was performed, and three days later thoracotomy with drainage of the pleural cavity and jejunostomy were carried out. Three weeks after the jejunostomy a further thoracotomy was performed, and the ruptured oesophagus was repaired by oesophago-gastroplasty. As a result of this experience, Moore and Murphy suggested that drainage of the pleural cavity, together with jejunostomy, antibiotics, and stopping oral intake, allowed most ruptures of the oesophagus to heal. They questioned the wisdom of attempting oesophageal repair in patients such as their own-in a desperate general condition and 72 hours after the rupture had occurred. Recovery after spontaneous rupture of the oesophagus also occurred in a patient reported by Frink (1947). In this patient repeated thoracenteses were done, followed three weeks later by a rib resection with drainage. Two months after admission the oesophageal tear had healed. Lynch (1949) reported a patient in whom an oesophageal perforation was diagnosed 60 hours after the onset of rupture. Temporary drainage of the pleural cavity with an intercostal tube was instituted and followed later by open drainage of a mediastinal abscess and empyema. The patient was acutely ill for three months before he recovered.

In recent years, with increasing recognition of the clinical picture, spontaneous rupture of the oesophagus has often been diagnosed correctly soon after onset, and many operative closures of the oesophageal tears have been carried out with survival of the patient. Barrett (1947), Olsen and Clagett (1947), Carter, Alrich, and Drash (1951), Samson (1951), Anderson (1952), and Borrie (1955) are among many who have reported such operative cures. These successes have prompted a number of reports analysing the results of different methods of treatment for spontaneous rupture of the oesophagus. Thus Samson (1951) found that, of 15 cases treated by repair of the oesophageal tear, 10 survived. On the other hand, eight of 15 patients survived when treated only by drainage of the pleural cavity. In the latter group, the post-operative complications were greater and the hospital stay was longer than for those who underwent immediate repair. A similar analysis of 50 cases was carried out by Anderson (1957). With thoracotomy and suture of the ruptured oesophagus, 14 patients survived and five died. Of the patients treated only by surgical drainage, one survived and eight succumbed, while none of 22 patients survived without surgical treatment. In a more recent review (Derbes and Mitchell, 1956) of 55 patients, immediate repair of the 
ruptured oesophagus was performed in 35 . Of these, $25(71 \%)$ survived. In $10(28 \%)$ patients, however, the oesophageal repair broke down, resulting in a fistulous communication with the mediastinum and pleural cavity. Of the remaining 20 patients in whom only drainage of the thoracic cavity was performed, $10(50 \%)$ survived.

From the above selected experience indications emerge for the treatment of spontaneous oesophageal rupture.

If seen and diagnosed early, the treatment of choice and the one providing the highest survival rate is thoracotomy with the repair of the oesophageal tear. The presence of shock should not deter the surgeon from operating as soon as resuscitative methods have been instituted. The anaesthetic allows full oxygenation of the patient and provides relief from pain, while thoracotomy relieves tension pneumothorax and allows reexpansion of the lung. Together with blood replacement, these measures soon lead to a marked improvement in the patient's condition.

When the patient is first seen or correctly diagnosed 48 hours or later after the onset and a chest radiograph shows a fluid collection in the mediastinum or pleural cavity, a thoracotomy should be done and the pleural cavity emptied and drained. Borrie (1955) maintains, and many would agree, that it would be an undoubted folly to attempt to free and suture the oesophagus in the presence of a fully established empyema. In any case, oesophageal repair at this stage is almost certainly doomed to break down later and is therefore both futile and immensely time-consuming.

For patients diagnosed two or three days after rupture, in good general condition and without evidence of increasing mediastinal abscess or empyema, as happened in the patient here reported, operation is unnecessary. Such rare individuals have sustained a small oesophageal tear which fails to rupture the mediastinal pleura and therefore does not produce pleural contamination.

\section{SUMMARY}

A case is reported illustrating the rare event of a spontaneous cure following spontaneous rupture of the oesophagus.

An outline is given of the clinical features of spontaneous rupture of the oesophagus, which is becoming increasingly well recognized.
The indications for and results of various $\stackrel{\overrightarrow{\vec{D}}}{\rightarrow}$ methods of treatment used in spontaneous oeso- $\bar{C}$ phageal rupture are discussed; in particular, a small but definite place for non-operative treatment is suggested.

Thanks are due to Professor D. S. Chapman, Head $\vec{\circ}$ of the Department of Surgery, University of Natal, $\overrightarrow{\vec{\omega}}$ for valuable criticism; to Dr. R. M. A. Nupen, Acting Medical Superintendent, King Edward VIII Hospital, Durban, for permission to publish the case $x$ report; to Mr. C. R. Brand, Senior Technician, 곤 Department of Surgery, for the photographs; and to iv Mrs. S. E. Harkcom, Secretary, Department of Surgery, for typing the manuscript.

\section{REFERENCES}

Anderson, R. L. (1952). Rupture of the esophagus. J. thorac. Surg., 24, 39.

(1957). Spontaneous rupture of the esophagus. Amer. J. Surg., চ 93, 282.

Barrett, N. R. (1946). Spontaneous perforation of the oesophagus. Review of the literature and report of three new cases. Thorax, 1, O 48.

(1947). Report of a case of spontaneous perforation of the oesophagus successfully treated by operation. Brit. J. Surg., 35, 216.

Boerhaave, H. (1724). Atrocis, nec descripti prius, morbi historia. ‡ Secundum medicae artis leges conscripta. Lugduni Batavorum $\mathbb{D}$ (quoted by Barrett, 1946).

Borrie, J. (1955). Spontaneous rupture of the oesophagus. Brit. med. J., $\overrightarrow{\vec{\sigma}}$ $1,23$.

Carter, J. P., Alrich, E. M., and Drash, E. C. (1951). Spontaneous rupture of the esophagus. Surgery, 30, 500.

Derbes, V. J., and Mitchell, R. E., jr. (1956). Rupture of the esophagus. Ibid, 39,688 .

Eliason, E. L., and Welty, R. F. (1946). Spontaneous rupture of the esophagus. Surg. Gynec. Obstet., 83, 234.

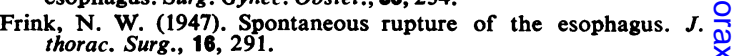

Graham, E. A. (1944). Editorial comment. Yearbook of General Surgery, p. 382.

Kinsella, T. J., Morse, R. W., and Hertzog, A. J. (1948). Spontaneous rupture of the esophagus. J. thorac. Surg., 17, 613 .

Lynch, J. P. (1949). Spontaneous perforation of the esophagus. New Engl.J. Med., 241, 395

Mallory, K. G., and Weiss, S. (1929). Hemorrhages from lacerations $O$ of the cardiac orifice of the stomach due to vomiting. Amer. J. med. Sci., 178, 506.

Meyer, J. (1858). Ueber Zerreissung der Spieseröhre. Med. Zeitung, n.s., 1, 189, 195 (quoted by Tesler and Eisenberg, 1963).

Moore, J. A., and Murphy, J. D. (1948). Spontaneous rupture of the N esophagus. J. thorac. Surg., 17, 632.

Moynihan, N. H. (1954). Pressure perforation and rupture of the oesophagus. Lancet, $\mathbf{2}, 728$.

Olsen, A. M., and Clagett, O. T. (1947). Spontaneous rupture of the esophagus. Postgrad. Med., 2, 417.

Priviteri, C. A., and Gay, B. B.,jr. (1951). Spontaneous rupture of the esophagus. Radiology, $57,48$.

Samson, P. C. (1951). Postemetic rupture of the esophagus. Surg. Gynec. Obstet., $93,221$.

Storey, C. F. (1962). Acquired Surgical Lesions of the Esophagus. \& Chap. 9, p. 156. Thomas, Springfield, Illinois.

Tesler, M. A., and Eisenberg, M. M. (1963). Collective Review; $T$ Spontaneous esophageal rupture. Int. Abstr. Surg., $117,1$. 